\title{
Polymorphisms in ANGPTL4 link triglycerides with CAD
}

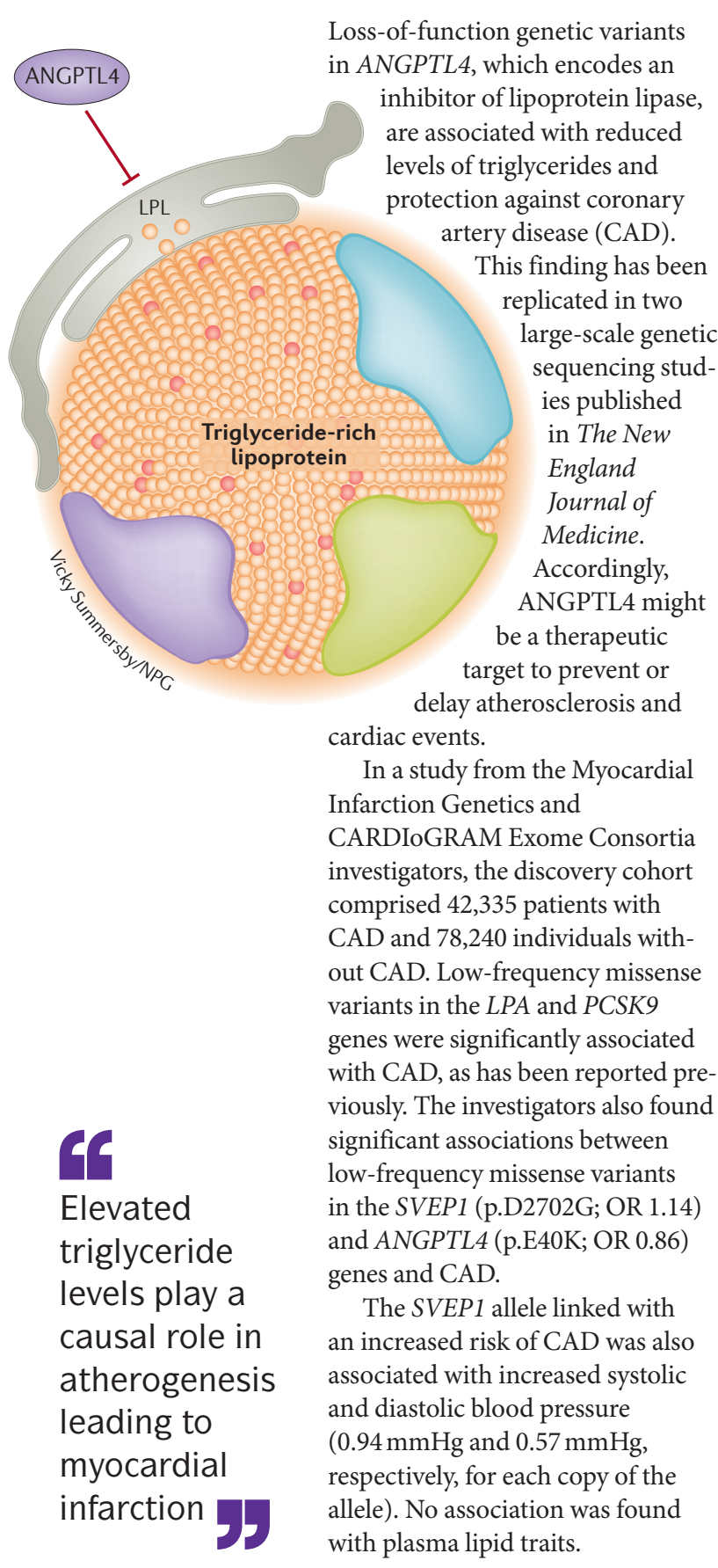

ANGPTL4 encodes angiopoietinlike 4 , a protein that normally suppresses the activity of endothelialbound lipoprotein lipase, the main enzyme that hydrolyses plasma triglyceride-rich lipoproteins, such as chylomicrons. Triglyceride levels were 35\% lower, and HDL-cholesterol levels were higher, in individuals with the loss-of-function ANGPTL4 allele than in those without. Individuals with the loss-of-function allele were at significantly reduced risk of myocardial infarction (OR 0.47).

Given that ANGPTL4 inhibits lipoprotein lipase, the investigators searched for mutations in LPL. A loss-of-function missense variant (p.D36N) was found to be associated with an increased risk of CAD (OR 1.13), and a gain-of-function nonsense variant (p.S447*) was found to be protective against CAD (OR 0.94). Nathan Stitziel, lead investigator of the study, comments that "these findings ... highlight the lipoprotein lipase pathway as a therapeutic target for preventing CAD”.

In a study from the Regeneron Genetics Center and Regeneron Pharmaceuticals, investigators tested for an association between CAD and ANGPTL4 in 10,552 patients with CAD and 29,223 individuals without CAD. The study group included 1,661 heterozygotes and 17 homozygotes for the $\mathrm{pE} 40 \mathrm{~K}$ variant, and 75 individuals with 13 other monoallelic inactivating mutations in ANGPTL4. In carriers of the p.E40K variant, triglyceride levels were $13 \%$ lower, HDL-cholesterol levels were $7 \%$ higher, and the risk of CAD was lower (OR 0.81) than in noncarriers. Similar associations were observed in carriers of other inactivating mutations.
Frederick Dewey, first author on the trial report, concludes that the findings "firmly establish a protective role for loss of ANGPTL4 function in CAD". The investigators are "continuing to explore the therapeutic opportunities afforded by targeting inhibitors of lipoprotein lipase, including ANGPTL3, for which Regeneron currently has an antibody in clinical development".

In preliminary studies by Dewey and colleagues, inhibition of Angptl4 using a monoclonal antibody in mice and monkeys successfully reduced triglyceride levels; however, an association was observed between this treatment and pathological accumulation of lipids in mesenteric lymph nodes.

Robert Hegele, from Robarts Research Institute, London, Ontario, Canada, and who was not involved in either research group, agrees that "both projects support the evolving narrative that elevated triglyceride levels play a causal role in atherogenesis leading to myocardial infarction". However, according to Hegele, the antibody experiments by Dewey et al. suggest that "ANGPTL4 biology is more complex than the population genetics would indicate; careful evaluation is needed before treatments targeting ANGPTL4 are 'ready for prime time' in humans".

Gregory B. Lim

ORIGINAL ARTICLES Myocardial Infarction Genetics and CARDloGRAM Exome Consortia Investigators. Coding variation in ANGPTL4, LPL, and SVEP1 and the risk of coronary disease. N.Engl.J. Med. http://dx.doi.org/10.1056/ NEIMoa1507652 | Dewey, F. E. et al. Inactivating variants in ANGPTL4 and risk of coronary artery disease. N. Engl.J. Med. http://dx.doi.org/10.1056/ NEJMoa1510926

FURTHER READING Watts, G. F. et al. Demystifying the management of hypertriglyceridaemia. Nat. Rev. Cardiol. 10, 648-661 (2013) 